\title{
Shape evolution and scaling analysis of soluble cylinders in dissolutive flow
}

Cite as: Phys. Fluids 32, 102103 (2020); https://doi.org/10.1063/5.0021805

Submitted: 15 July 2020 . Accepted: 15 September 2020 . Published Online: 01 October 2020

Qing Miao (苗青), (D) Quanzi Yuan (袁泉子), and (iD) Ya-Pu Zhao (赵亚溥)

\section{COLLECTIONS}

Paper published as part of the special topic on Recent Advances in Theory, Simulations, and Experiments on Multiphase FlowsMPF2020
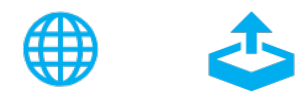

\section{ARTICLES YOU MAY BE INTERESTED IN}

Theoretical analysis for flattening of a rising bubble in a Hele-Shaw cell

Physics of Fluids 32, 092102 (2020); https://doi.org/10.1063/5.0016080

Finger instability of oscillating liquid-liquid interface in radial Hele-Shaw cell

Physics of Fluids 32, 102102 (2020); https://doi.org/10.1063/5.0018541

Locomotion of a rotating cylinder pair with periodic gaits at low Reynolds numbers

Physics of Fluids 32, 103102 (2020); https://doi.org/10.1063/5.0022681

\section{Physics of Fluids GALLERY OF GOVERS}

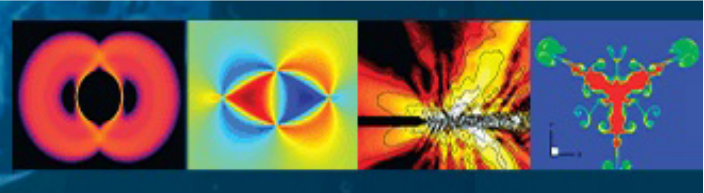




\title{
Shape evolution and scaling analysis of soluble cylinders in dissolutive flow
}

\author{
Cite as: Phys. Fluids 32, 102103 (2020); doi: 10.1063/5.0021805 \\ Submitted: 15 July 2020 - Accepted: 15 September 2020 • \\ Published Online: 1 October 2020
}

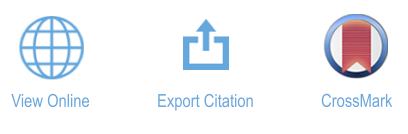

Qing Miao (苗青), Quanzi Yuan (袁泉子), (D) and Ya-Pu Zhao (赵亚溥) ${ }^{a)}$

\begin{abstract}
AFFILIATIONS
State Key Laboratory of Nonlinear Mechanics, Institute of Mechanics, Chinese Academy of Sciences, Beijing 100190, People's Republic of China and School of Engineering Science, University of Chinese Academy of Sciences, Beijing 100049, People's Republic of China
\end{abstract}

\begin{abstract}
Note: This paper is part of the Special Topic, Recent Advances in Theory, Simulations, and Experiments on Multiphase Flows. a) Authors to whom correspondence should be addressed: yuanquanzi@lnm.imech.ac.cn and yzhao@imech.ac.cn
\end{abstract}

\begin{abstract}
The evolution of solid shapes in dissolutive flows is studied using molecular dynamics simulations. The final self-similar structures of the solid are distinct under the convection- and diffusion-dominated conditions. Introducing a dimensionless number, $D s$, allows characterizing the relative influence of convection and diffusion on the final structure. When convection dominates, the convergent shape of the solid is approximately triangular, while the solid is more likely to be sculptured into a cylinder when diffusion dominates. There is a critical value of $D s$ that controls the transition between convection- and diffusion-dominated cases. However, the convergent shapes are insensitive to their initial states due to the solid assembly at the nanoscale. Furthermore, we discuss the influences of solid dissolution and assembly on the liquid density along different directions and provide fitting curves for the theoretical density distribution as explained from the Smoluchowski equation. Finally, the scaling laws are constructed to quantify the solid evolution, which can analytically forecast the shape evolution under different dominant factors. We believe that these findings provide theoretical support for structure optimization and industrial applications.
\end{abstract}

Published under license by AIP Publishing. https://doi.org/10.1063/5.0021805

\section{INTRODUCTION}

Shape evolution in dissolutive flow is an intricate dynamic problem due to coupling between flowing fluids and receding solid boundaries. However, its rising interest in industrial applications ${ }^{1}$ and academic research ${ }^{2}$ has culminated in significant attention. For instance, the remarkable classical Stephen problem, which focuses on phase changes at an interface, has a significant influence in both physics $^{3,4}$ and mathematics. ${ }^{5,6}$ The diffusion boundary condition at the moving contact line also provides a new method to settle the Huh-Scriven paradox. ${ }^{7}$ Besides, with the rapid development of micro- and nanotechnology, the system of soluble nanoparticles plays increasingly important roles in biological problems and pharmaceutical preparations; if the dissolution of the drug can be controlled, the drug utilization rate will be improved, which requires focusing on interface behaviors and has stimulated many studies. Moreover, in shale gas exploitation, if the rock can be dissolved simultaneously, when a supercritical fluid flows through a porous channel, it will increase reservoir connectivity and also encourage energy efficiency. ${ }^{10}$ The surface morphologies caused by the dissolutive flow are also crucial when analyzing geological landscapes and pattern evolutions, ${ }^{11,12}$ chemical and pharmaceutical efficiency, ${ }^{13,14}$ metal alloy formation, ${ }^{15,16}$ and other fields. ${ }^{17}$

Over the past few decades, some laboratory experiments at the continuum scale have considered sculpted shapes from solids in fast-flowing fluids. ${ }^{12,18-21}$ Scaling and free-streamline analyses of hard candy and clay in fast-flowing water under different initial shapes have been performed. ${ }^{12,20}$ The results show that solid morphologies eventually converge to different self-similar shapes under various solubilities and flow velocities. They also analyzed the physical mechanisms during the dissolution process. These analyses provide an intuitive understanding of the physical mechanisms for the self-similar evolution of solids at the macroscale.

In contrast, the microscopic perception of solid evolution that considers the factors of size, diffusion, interatomic forces, and solid 
assembly in dissolutive flows is still out of reach. Even if dramatic breakthroughs were made when considering nanoscale fluid flowing around insoluble solid, ${ }^{22,24}$ it would still be unfeasible to experimentally consider the dissolution effects and track the changes to fluid characteristics and soluble solid morphologies. ${ }^{25-27}$ Thus, numerical simulations are the primary means of investigation. We previously simulated dissolutive flows in nanochannels with focus on changes in the fluid structure. ${ }^{28}$ Nevertheless, further analyses and studies are needed when discussing the receding boundary and solid evolution.

This paper highlights the behaviors of soluble solids with different initial configurations in flowing fluids using molecular dynamics (MD) simulations. The evolution processes of the interface morphology, flow field, fluid density distribution, and dissolution rate at different fluid velocities and solubilities are given from a microscopic perspective. Thus, we quantitatively explore the scaling laws of solid regions with time. These findings help further understand the mechanisms and characteristics of dissolutive flow and their processes in nature. We believe that this work provides theoretical support for structure optimization and industrial applications.

\section{MOLECULAR DYNAMICS SIMULATIONS}

As MD simulations are widely used to study interface problems, ${ }^{29,30}$ fluid flowing around the cylinder and rectangular prism is simulated using the LAMMPS package. Our proposed model follows the concept of fluid flowing around insoluble bluff bodies ${ }^{23,31}$ but changes the solubility of the solid atoms. The geometry of our model is illustrated in Figs. 1(a) and 1(b), where the atoms in red are bluff bodies whose central particles are fixed and the liquid in blue employs the extended single point charge water model. ${ }^{32,33}$ Simulations of the fluid flow over the insoluble solid are performed as a control group. ${ }^{34} \sim 80000$ atoms are contained in the simulation systems. Since it focuses on two-dimensional simulations, the simulation box is about $260 \times 20 \times 200 \AA^{3}$ with periodic boundary conditions. Two different initial configurations, cylinder and rectangular prism, are employed, as illustrated in Figs. 1(a) and 1(b). The diameter of the central solid is set as $60 \AA$.

The interactions between particles consist of two parts,

$$
E_{i j}=4 \varepsilon_{i j}\left[\left(\sigma_{i j} / r_{i j}\right)^{12}-\left(\sigma_{i j} / r_{i j}\right)^{6}\right]+q_{i} q_{j} / \varepsilon_{0} r_{i j} .
$$

First, the van der Waals interactions between particles follow the classical Lennard-Jones potential ${ }^{33}$ in which $\varepsilon_{i j}, r_{i j}$, and $\sigma_{i j}$ indicate the potential depth, distance, and effective molecular diameter between two atoms $i$ and $j$, respectively. ${ }^{28}$ Without changing the wettability $\varepsilon_{i j},{ }^{35,36}$ we achieve variations in the solubility by altering the intrinsic properties of the solid particles. The dimensionless $\varepsilon=\varepsilon_{s l} / \varepsilon_{s s}$ is employed to characterize the solubility of solid atoms. A larger $\varepsilon$ indicates easier dissolution of the solid. Besides, the specific values of $\sigma_{i j}$ and $\varepsilon_{i j}$ when $\varepsilon=1.250$ are given in the supplementary material. Second, the Coulomb potential between liquid particles is calculated as $E_{i j}=q_{i} q_{j} / \varepsilon_{0} r_{i j}$, where $q_{\mathrm{O}}=-0.8476 \mathrm{e}$ and $q_{\mathrm{H}}=+0.4238 \mathrm{e}$ are the charges for oxygen and hydrogen atoms, respectively, and $\varepsilon_{0}$ is the electrostatic constant.

After relaxation in the isothermal-isobaric (NPT) ensembles for $100 \mathrm{ps}$, the simulations are run as canonical (NVT) ensembles, and the Nose-Hoover thermostat is used to maintain the temperature at $350 \mathrm{~K}$. Interactions between particles are truncated at $1 \mathrm{~nm}$. The velocity-Verlet algorithm is used to solve the particle equations of motion with a time step of $1 \mathrm{fs}$, while the statistical time step is $10^{5} \mathrm{fs}$. A total of 100-500 statistical time steps, corresponding to the inflow velocity of $1 \mathrm{~m} / \mathrm{s}-0.1 \mathrm{~m} / \mathrm{s}$, are simulated. As liquid exits downstream in the $x$-direction, it re-enters the upstream with a reassigned velocity and no dissolved particles. A transverse force field, whose magnitude is determined empirically based on the previous literature, ${ }^{31}$ is applied to maintain the flow. Previous experiments and simulations show that the convection velocity profoundly affects the dissolution process. ${ }^{12,20}$ Thus, simulations under different velocities are performed $(u=0.1 \mathrm{~m} / \mathrm{s}, 0.5 \mathrm{~m} / \mathrm{s}, 1.0 \mathrm{~m} / \mathrm{s})$. The different impact factors, including the solubility, initial geometry, and fluid velocity, (a)

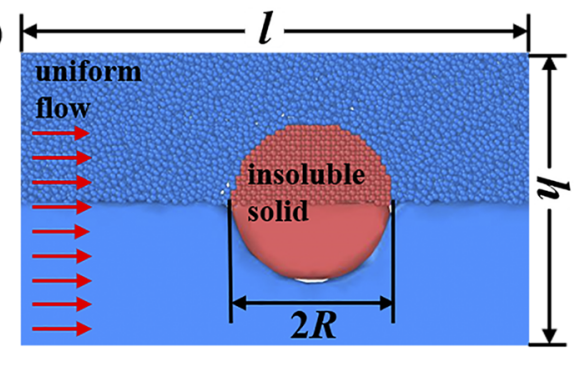

(c)

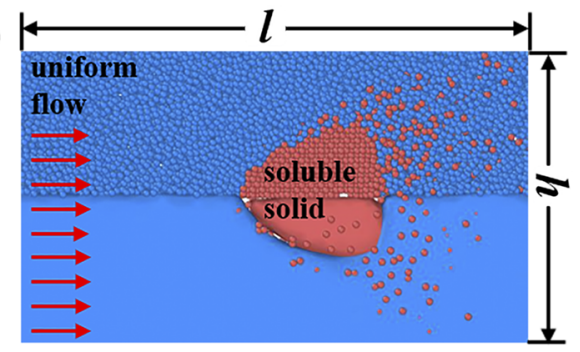

(b)

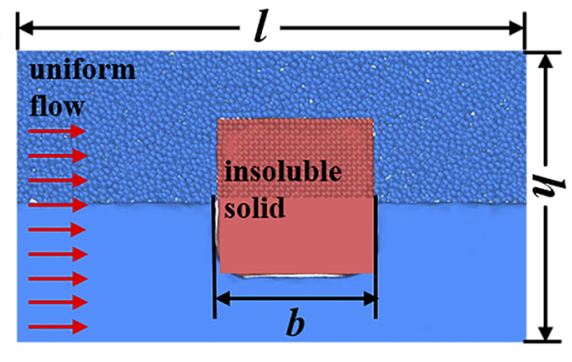

(d)

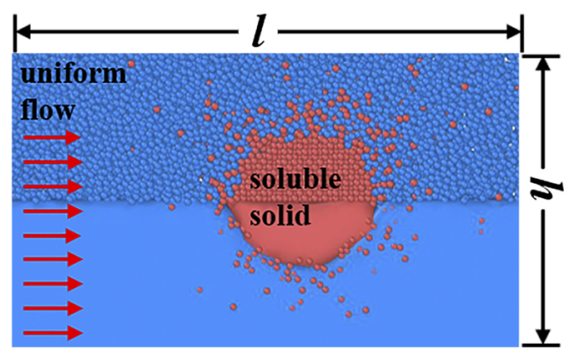

FIG. 1. Simulation domains. The inlet flow is controlled as uniform with velocity $U_{0}$ labeled as the red arrows. Illustrations of liquid flows over (a) an insoluble cylinder and (b) an insoluble rectangular prism. The convergent shapes of the solid when (c) the convection dominates and (d) diffusion dominates. The red and blue parts depict the solid and liquid, respectively. 
TABLE I. Different simulation conditions.

\begin{tabular}{|c|c|c|c|c|c|c|}
\hline \multirow[b]{2}{*}{$D s=\frac{u / U}{\varepsilon-1}$} & \multicolumn{2}{|c|}{$\varepsilon=0.1$} & \multirow{2}{*}{$\begin{array}{l}\varepsilon=1.111 \\
\begin{array}{l}\text { Circular } \\
\text { cylinder }\end{array}\end{array}$} & \multicolumn{2}{|c|}{$\varepsilon=1.250$} & \multirow{2}{*}{$\begin{array}{l}\varepsilon=1.429 \\
\begin{array}{l}\text { Circular } \\
\text { cylinder }\end{array}\end{array}$} \\
\hline & $\begin{array}{l}\text { Circular } \\
\text { cylinder }\end{array}$ & $\begin{array}{l}\text { Square } \\
\text { cylinder }\end{array}$ & & $\begin{array}{l}\text { Circular } \\
\text { cylinder }\end{array}$ & $\begin{array}{l}\text { Square } \\
\text { cylinder }\end{array}$ & \\
\hline$u / U=0.1$ & -0.111 & 0.111 & 0.901 & 0.400 & 0.400 & 0.233 \\
\hline$u / U=0.5$ & -0.555 & 0.555 & 4.505 & 2.000 & 2.000 & 1.166 \\
\hline$u / U=1.0$ & -1.111 & 1.111 & 9.009 & 4.000 & 4.000 & 2.331 \\
\hline
\end{tabular}

are varied to obtain 18 different simulation conditions, as listed in Table I. The main results are shown in the schematic of Figs. 1(c) and 1 (d).

\section{RESULTS AND DISCUSSION}

\section{A. Evolution of solid morphology}

\section{Shape evolution}

Figure 2 shows the evolution of the solid morphology in the MD simulations. Previous macroscopic results show that the final morphologies of the soluble solids differ when the dominant actions are convection ${ }^{12}$ and diffusion. ${ }^{20}$ The main difference ${ }^{20}$ is the angle formed at the stagnation points when the backs are both flat, and the self-similar shapes occur when $R e \sim 10^{4}$. In atomic simulations, we find that a self-similar evolution of the solid is observed when $R e$ is less than 1. Moreover, macroscopic studies have found that even in the absence of fluid flow, the soluble solid's back is nearly flat due to gravitational effects. ${ }^{37}$ However, in microscopic cases, the planar structure of the back surface cannot be observed without fluid flow. Thus, additional microscopic details need to be considered. (a) $0<D s<1$

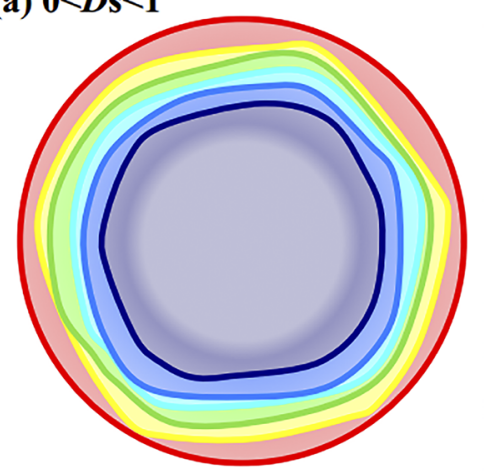

(c) $D s>1$

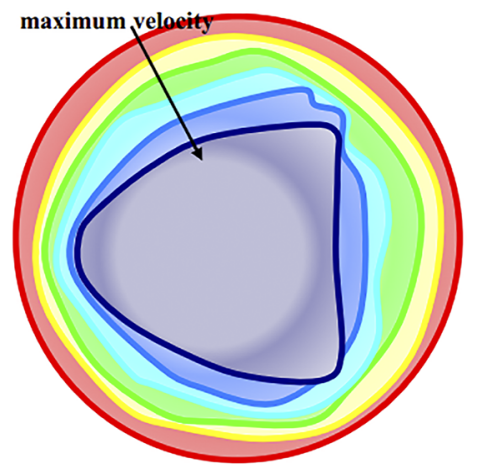

(b) $0<D s<1$
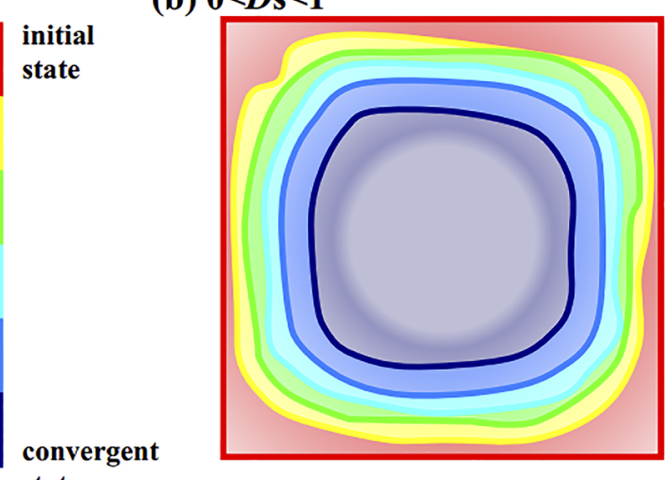

state

\section{(d) $D \mathrm{~s}>1$}

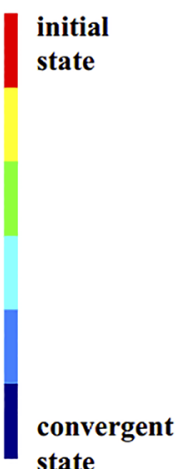

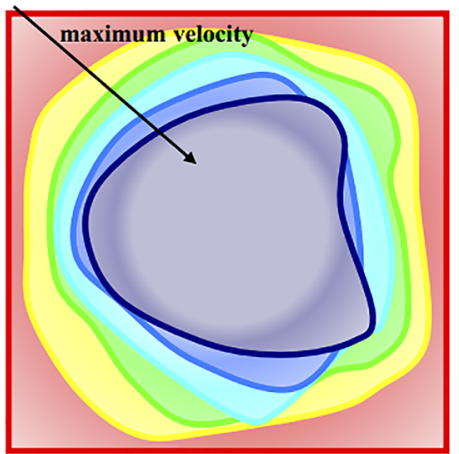

convergent

state

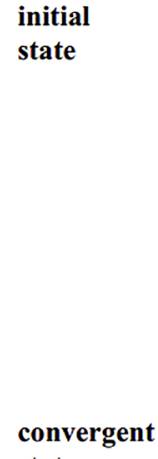

state

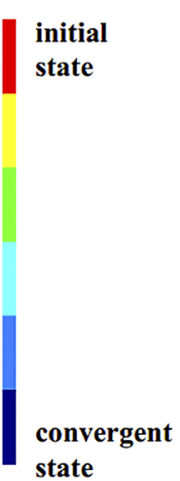

FIG. 2. Shape evolution of solid in dissolutive flow. The cross-sectional change is determined every 20 statistical steps. (a) and (c) cylinders and (b) and (d) rectangular prisms over time. The arrows in (c) and (d) point roughly in the direction of steepest descent. 
In our simulations, the characteristic time of the fluid to bypass the cylinder is expressed as $\tau_{1}=R / u$. Considering the kinematic viscosity as the diffusion coefficient for momentum, the characteristic time for momentum diffusivity is $\tau_{2}=\rho R^{2} / \eta$. From the flow field in Fig. 5 , there is a slip boundary when the inflow velocity is $1 \mathrm{~m} / \mathrm{s}$. As discussed previously, ${ }^{38,39}$ the viscosity of water at the nanoscale is different from that at the macroscale so that viscosity is of the order of $10^{-5} \mathrm{~Pa}$ s when the velocity is nearly $1 \mathrm{~m} / \mathrm{s}$ at $350 \mathrm{~K}$. The detailed calculation of the viscosity is provided in the supplementary material. Introducing $U$ as the characteristic velocity with $R e \sim 1$ allows writing $\tau_{2}$ as $\tau_{2}=\rho R^{2} / \rho U R=R / U$. Thus, $\tau_{1} / \tau_{2}=u / U$, i.e., $u / U$ represents the ratio of the characteristic time of convection and momentum diffusivity, which reflects the convection effect. Moreover, $\left(\varepsilon_{s l}-\varepsilon_{s s}\right) / \varepsilon_{s s}=\varepsilon-1$ is the ratio of energy mentioned in Sec. II and can describe the diffusion capacity. To specify the system properties, we combine the dimensionless time and energy as a new dimensionless number $D s$, which is similar to the action that describes the inherent dynamics of physical systems. We define $D s$ as $D s=(u / U) /(\varepsilon-1)$, where $u$ is the convection velocity, $U$ is the characteristic velocity with $R e \sim 1$, and $\varepsilon$ is the dimensionless energy in our simulations. The solid is insoluble when $D s<0$. However, when $D s>0$ the dissolution process is gradually controlled by the inflow velocity with larger $D s$. As depicted in Fig. 2, when $0<D s<1$, the cylinder mostly retains its initial shape, and the rectangular prism gradually approaches a cylinder shape. Moreover, solids with different initial configurations eventually converge to form a uniform quasi-triangular prism when $D s>1$. In other words, the final solid shape is insensitive to the initial state.
We analyzed positional changes of the particles included in the final configuration, as shown in Fig. 3. Figures 3(a1) and 3(a2) show the final shapes of the solid, Figs. $3(\mathrm{c} 1-\mathrm{j} 1)$ and $3(\mathrm{c} 2-\mathrm{j} 2)$ give the evolution details, and Figs. 3(b1) and 3(b2) are the initial structures. It is seen that some of the atoms (yellow) first dissolve during the solid shape evolution and then self-assemble primarily at the two corners at the back of the triangle. Near the solid wall, the first layer of dissolved atoms is more likely to directly self-assemble. The second layer of atoms tends to slide along the solid wall, and the third layer of atoms flows along the flow field. Those different initial configurations converge to their corresponding final structures and obey different mechanisms, which necessitate quantitative calculations.

\section{Behavior of dissolved particles and dissolution rate along solid curves}

Figures 4(a)-4(d) show the detachment and attachment particles at the initial time with different inflow velocities under various initial configurations. As the two lines approach, the net number of dissolved particles decreases. When $D s>1$, there is a stagnation point in both the cylinder and rectangular prisms, as highlighted in Fig. 3. The stagnation points correspond to the back two vertices of the triangular prisms. In front of this point, the dissolution rate (see the inset of Fig. 4) is relatively high, and the two sides of the triangular prisms are formed. Behind this point, the fluid bypasses the cylinder and rotates toward the tail, as shown in Figs. 5(e)-5(h), which results in the back of the cross section evolving into a plane (back of the triangle). In particular, as indicated in the red circles of the inset of Fig. 4(c), there are two dissolution peaks at right angles for the cross section of rectangular prisms. It is understood

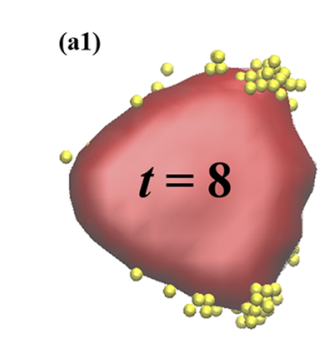

(c1)

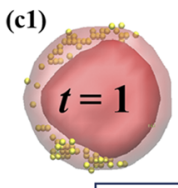

(d1)

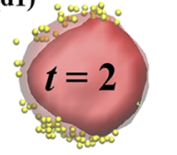

(e1)

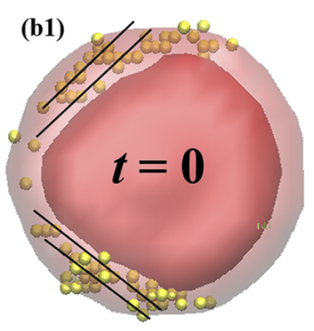

(f1)

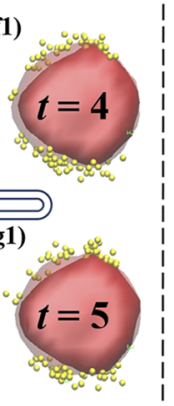

(a2)

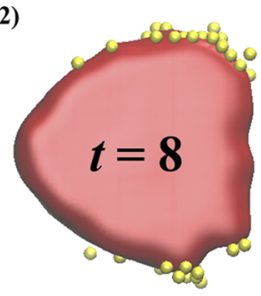

(c2)

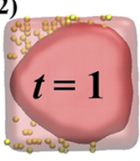

(d2) (b2)

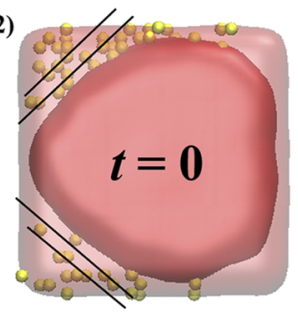

(f2)

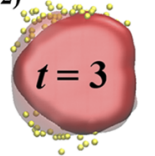

2)

(j2)

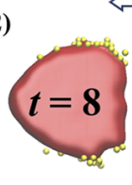

(i2)

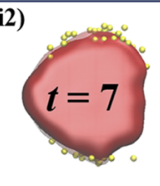

(h2)

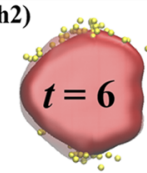

(g2)

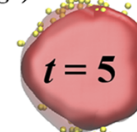

FIG. 3. Positional changes for the particles that constitute the final configuration when Ds $>1$. For the cylinder, (a1) and (b1) are the ultimate and initial configurations, respectively. (c1)-(j1) give the configurations at different times and position changes of the self-assembled atoms. For the rectangular prisms, (a2) and (b2) are the ultimate and initial configurations of the solid, respectively. (c2)-(j2) give the configurations at different times and position changes of the self-assembled atoms. The red part in the center of the solid corresponds to the final configuration, the red area on the outside shows the undissolved configuration at the current time, and the yellow dots are atoms that first dissolve and then self-assemble. 
(a)
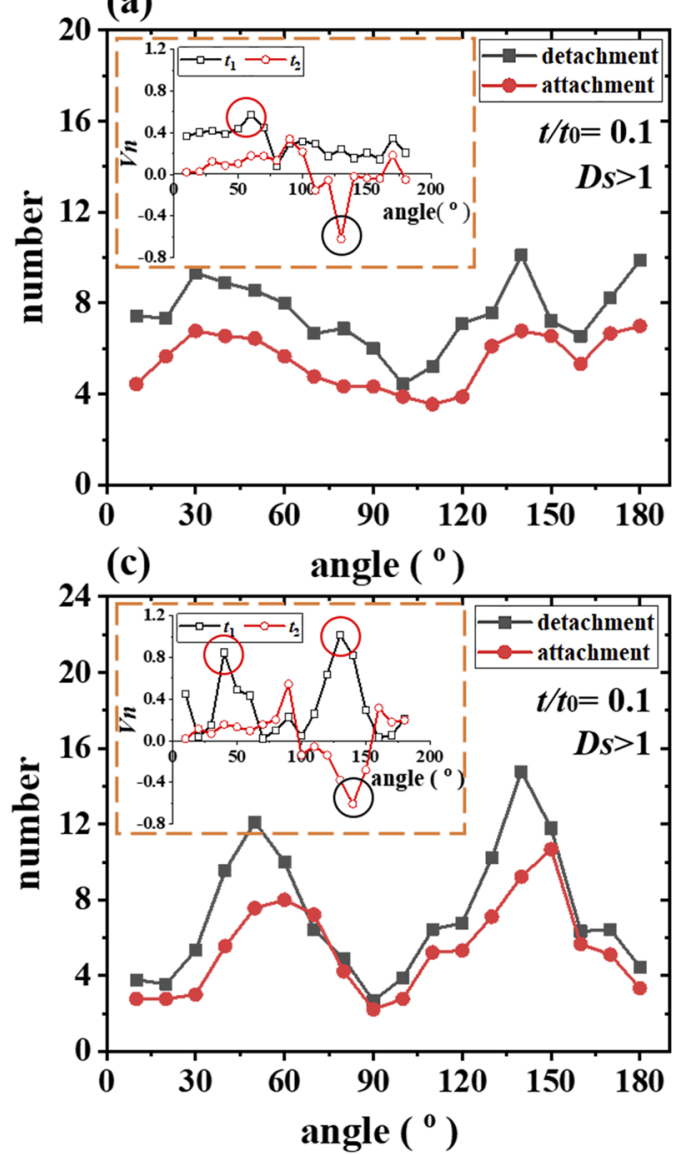

(b)
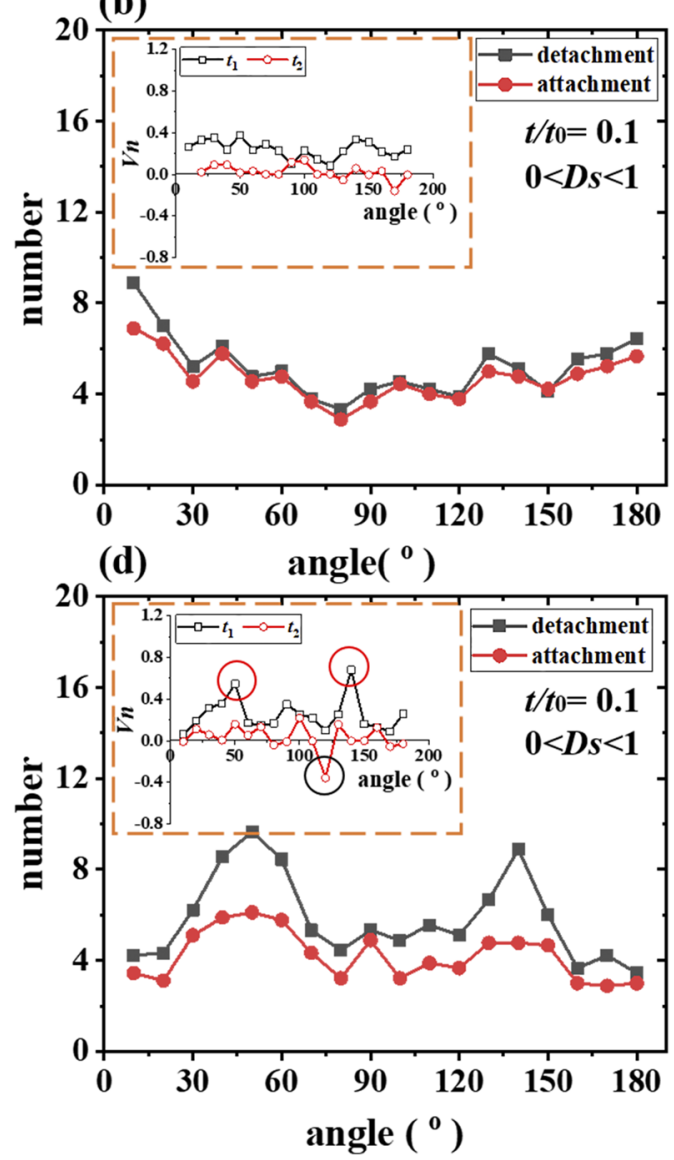

FIG. 4. The number of initial detachment and attachment atoms along the solid wall for the (a) and (b) cylinders and (c) and (d) rectangular prisms. The black lines with rectangles are the number of dissolved atoms, and the red lines with circles are the number of self-assembled atoms. The insets are the dissolution rate along the solid curves at different times $t_{1}$ and $t_{2}$. The black lines with hollow rectangles are the initial dissolution rates, and the red lines with hollow circles are the dissolution rate after some time. The points circled in red correspond to the positions with maximum velocity, and the points circled in black correspond to the self-assembly positions.

that these curvature singularities have lower dissolution activation energies and higher dissolution rates.

When $0<D s<1$, the fluid flux is negligible, as shown in Figs. 5(a)-5(d). The inset of Fig. 4(b) indicates that the dissolution rate is nearly the same along the solid wall for the cylinders so that the solid remains at its initial configuration. For rectangular prisms, there are still curvature singularities, and the solid cross section tends to become round. A self-similar structure is formed after some time, and the dissolution rate decreases, as shown in the red lines of the inset of Fig. 4. The detachment and attachment particle curves are nearly parallel, as shown in Fig. S2 of the supplementary material. The dissolution curves show that the position of the stagnation points changes over time, and the particles more easily self-assemble near these points. During the dissolutive flow, the behavior of the particles and the dissolution rate along the solid wall explain why the final configuration is insensitive to the initial shape. The self-assembly of particles varies greatly based on the properties of the liquid surrounding the solid, which is discussed later.

\section{B. Evolution of liquid density distribution}

The diffusion effect on the fluid structure is significant when the fluid flows around the soluble solid. The non-equilibrium MD simulations are used to analyze the density distributions of the fluid and water molecules along different directions. The area near the solid is divided into small grids, and the number densities of particles inside the different grids are based on statistical averages, as shown in Fig. 6. Typical insoluble and soluble solids for the circular cross section are selected as comparisons. When the solid is insoluble, a distinct high-density region in front of the solid is observed, which is consistent with the previous results. ${ }^{40}$ The density distribution of water molecules shows a peak near the solid surface, which is related to the fluid molecular structures at the nanoscale. ${ }^{41}$ The Smoluchowski formula derived from the Onsager principle ${ }^{42}$ allows 
(a)

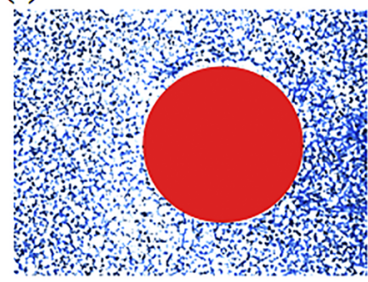

initial state (circle)

(e)

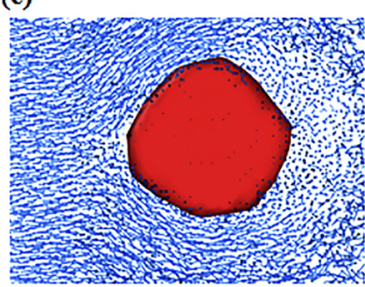

transitional state (square) (b)

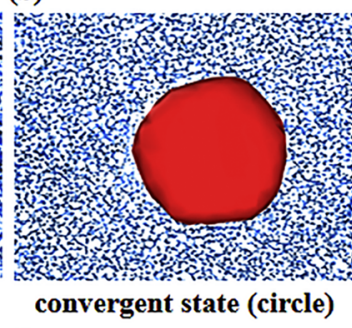

(f)

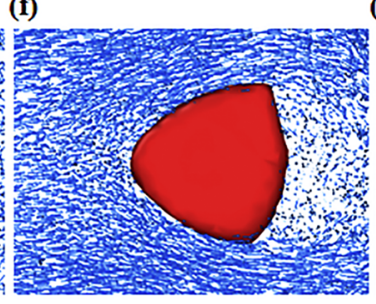

convergent state (square) (c)

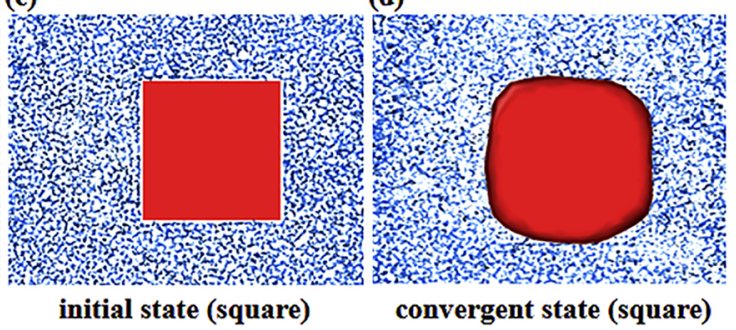

(g)

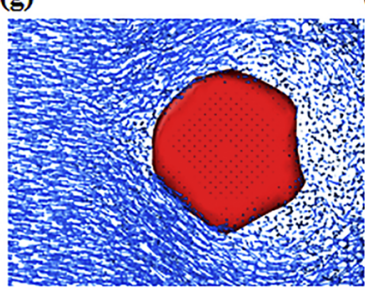

transitional state (square) (h)

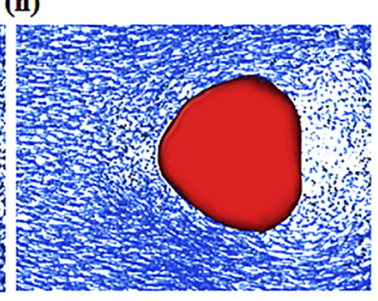

convergent state (square)

FIG. 5. Statistical average results of the flow field for the (a) initial and (b) convergent states for the flow fields with a cylinder when $0<D s<1$. The (c) initial and (d) convergent states for the flow fields with a rectangular prism when $0<D s<1$. The (e) transitional and (f) convergent states for the flow fields with a cylinder when $D s>1$. The $(\mathrm{g})$ transitional and $(\mathrm{h})$ convergent states for the flow fields with a rectangular prism when $D s>1$.

expressing the density distribution as

$$
\frac{\partial \rho}{\partial t}=D \frac{\partial}{\partial x}\left[\frac{\partial \rho}{\partial x}+\frac{\rho}{k_{B} T} \frac{\partial U^{\prime}}{\partial x}\right],
$$

where $\rho, T$, and $k_{\mathrm{B}}$ are the liquid number density, absolute temperature, and Boltzmann constant, respectively. Considering that the total potential energy corresponds to the disjoining pressure, ${ }^{42}$ the fluid density distribution is expressed in exponential form as $\rho(r)=\rho_{0} \exp \left[(-\omega) / k_{\mathrm{B}} T\right]$, where $\omega(r)$ is the total potential energy ${ }^{43}$ (a detailed derivation is given in the supplementary material). The exponential fitting lines are shown as red in Figs. 6(a1)-6(a5) and coincide with our previous results. ${ }^{2}$

The introduction of the dissolution affects the internal structure of the liquid, which further impacts its progress and makes this an intractable problem. We analyze the variations in the density distributions of the fluid and water molecules along different directions when the convection effect takes priority in the solid evolution. First, near the front stagnation point, the solid particles barely dissolve and the incoming liquid particles accumulate, which leads to liquid pressurization and an increased density. Therefore, as shown in Figs. 6(b1)-6(b5), increasing density peaks and decreasing the peak pitch to $0^{\circ}$ is intuitive. Along the other directions (from $45^{\circ}$ to $180^{\circ}$ ), the dissolution causes the disappearance of the liquid layered structure. Thus, the density can be expressed as

$$
\rho(r)=\rho_{0} \exp \left[(-\omega+G) / k_{\mathrm{B}} T\right]
$$

where $G$ is the dissolution energy.

For the insoluble cases, the various initial shapes result in different density distributions. Figure 6(a) shows the liquid density with an initial cylinder. In the rectangular prisms, there is no density peak along the $45^{\circ}$ and $135^{\circ}$ directions due to the curvature-related effects, and the density of water molecules continues to oscillate along the other directions. The dissolution is nearly homogeneous in all directions when $0<D s<1$ so that the layered structure of the fluid is uniformly disturbed, i.e., there is no density peak. The detailed density distributions in the above two cases are given in the supplementary material.

\section{Scaling analysis on the shape of the soluble solid}

The scaling laws of the solid shape with respect to time are explored to quantify the effects of the fluid velocity on the soluble solids. When $0<D s<1$, diffusion is the primary factor that controls the solid evolution. Thus, it is reasonable to use the Noyes-Whitney equation in our model as ${ }^{44}$

$$
v_{n}=D \frac{s\left(C_{s}-C\right)}{\delta_{c}},
$$

where $v_{n}, D, s, \delta_{c}, C_{s}$, and $C$ are the dissolution rate, diffusion coefficient, length of the solid-liquid contact line, thickness of the concentration boundary, saturation concentration, and instant concentration of the solute, respectively. The $D, \delta_{c}, C_{s}$, and $C$ are seen as constant with additional details given in the supplementary material. As $d A / d t \sim v_{n} s \sim s^{2} \sim A$, we obtain

$$
A \sim \exp \left(-k_{e} t / t_{0}\right),
$$

where $k_{e}$ is the elimination rate.

When $D s>1$, the dissolution is strengthened. Previous studies $^{20,21}$ have shown that the relationship between the dissolution rate, convection velocity, and characteristic length can be expressed as $v_{n} \sim \sqrt{U / s}$ when the Navier-Stokes equation is solved using the 
(a-1)

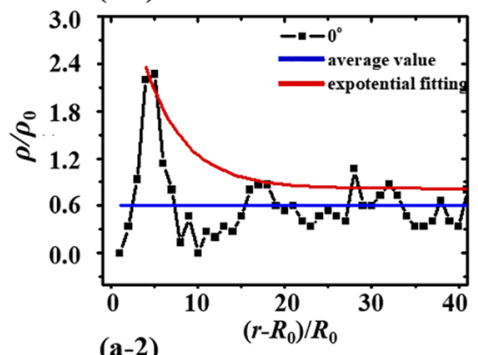

(a-2)

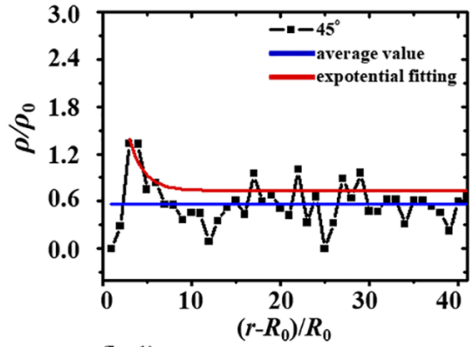

(b-1)

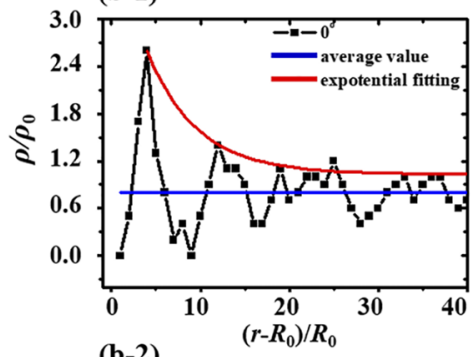

(b-2)

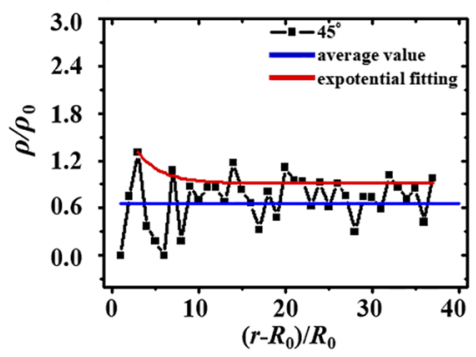

(a)

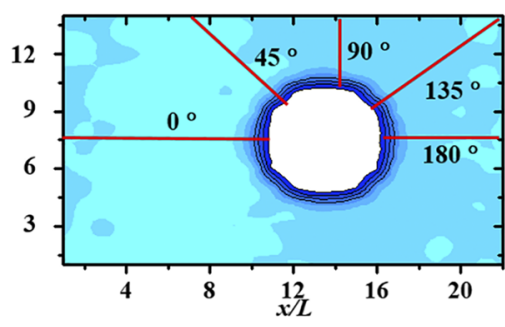

(a-3)

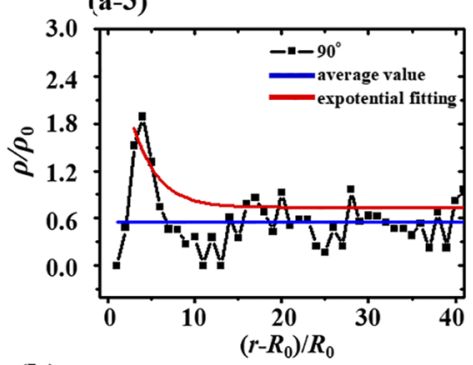

(b)

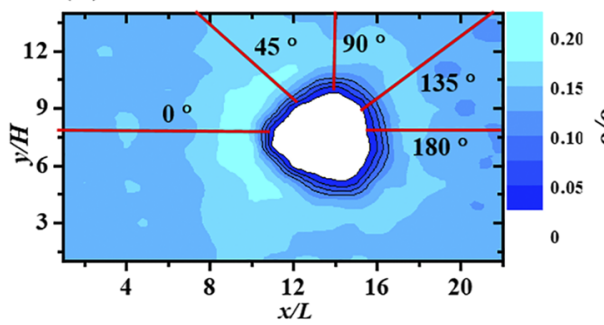

(b-3)

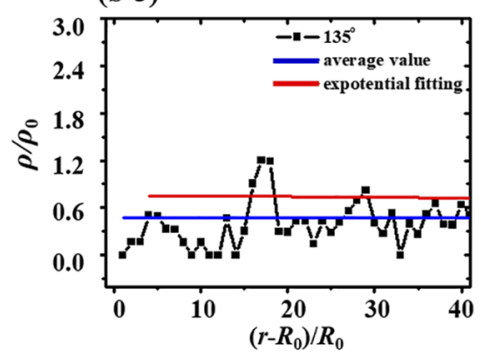

(a-5)

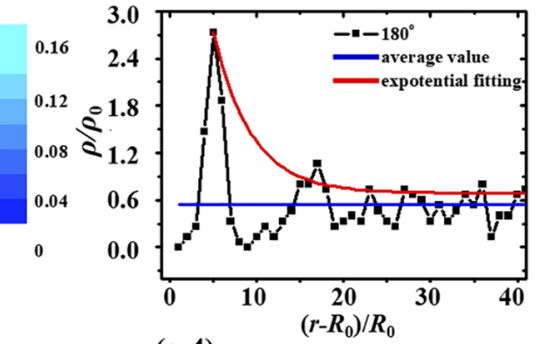

(a-4)

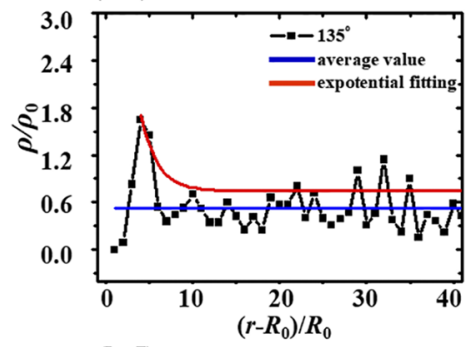

(b-5)

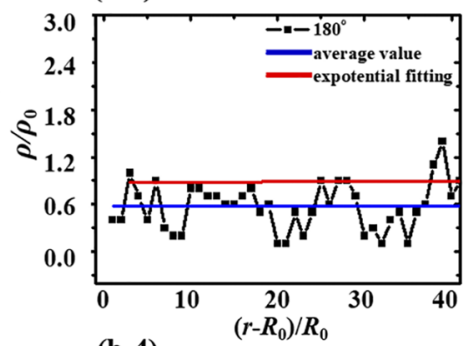

(b-4)

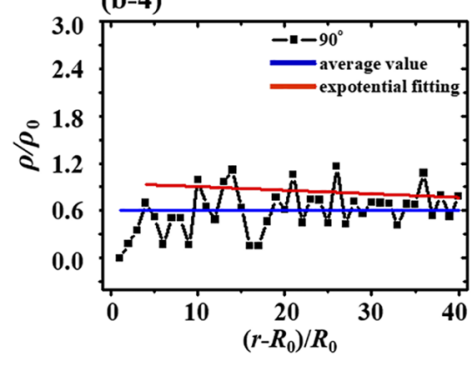

FIG. 6. Liquid density distribution. (a) Fluid density around the cylinders when solid particles are insoluble and (b) the density distribution of the surrounding fluid when the central solid shape converges to an approximately triangular prism. The light blue area represents higher densities, which gradually decrease for darker shades. The (a1)-(a5) and (b1)-(b5), respectively, correspond to the density distribution along the $0^{\circ}, 45^{\circ}, 90^{\circ}, 135^{\circ}$, and $180^{\circ}$ directions when the solid is insoluble and soluble, respectively. The red lines are the exponential curve fits of the density peak, the blues lines are the average of the fluid density, and the black lines with rectangles are the statistical results.

similarity solution. There is also a momentum and concentration boundary layer near the solid wall, as shown in Fig. S5. Thus, previous results ${ }^{28}$ can be referenced to understand the relationship between the dissolution rate and boundary length. As $d A / d t \sim v_{n} \mathcal{S} \sim \sqrt{\mathcal{S}} \sim A^{1 / 4}$, the scale relationship of the dissolved area with time satisfies the following expression:

$$
A \sim\left(1-t / t_{0}\right)^{4 / 3}
$$

where $t_{0}$ is the characteristic time when the solid disappears. When the convection velocity and dissolution rate are of the same order, the solid evolution over time is between the exponential and the $4 / 3$ scaling relation. The results are shown in Fig. 7 , where $D s=4,2$, and 0.4 , under different initial configurations, showing good agreement between the derived theory and simulations. The other cases listed in Table I also follow the same scaling laws. 
(a)
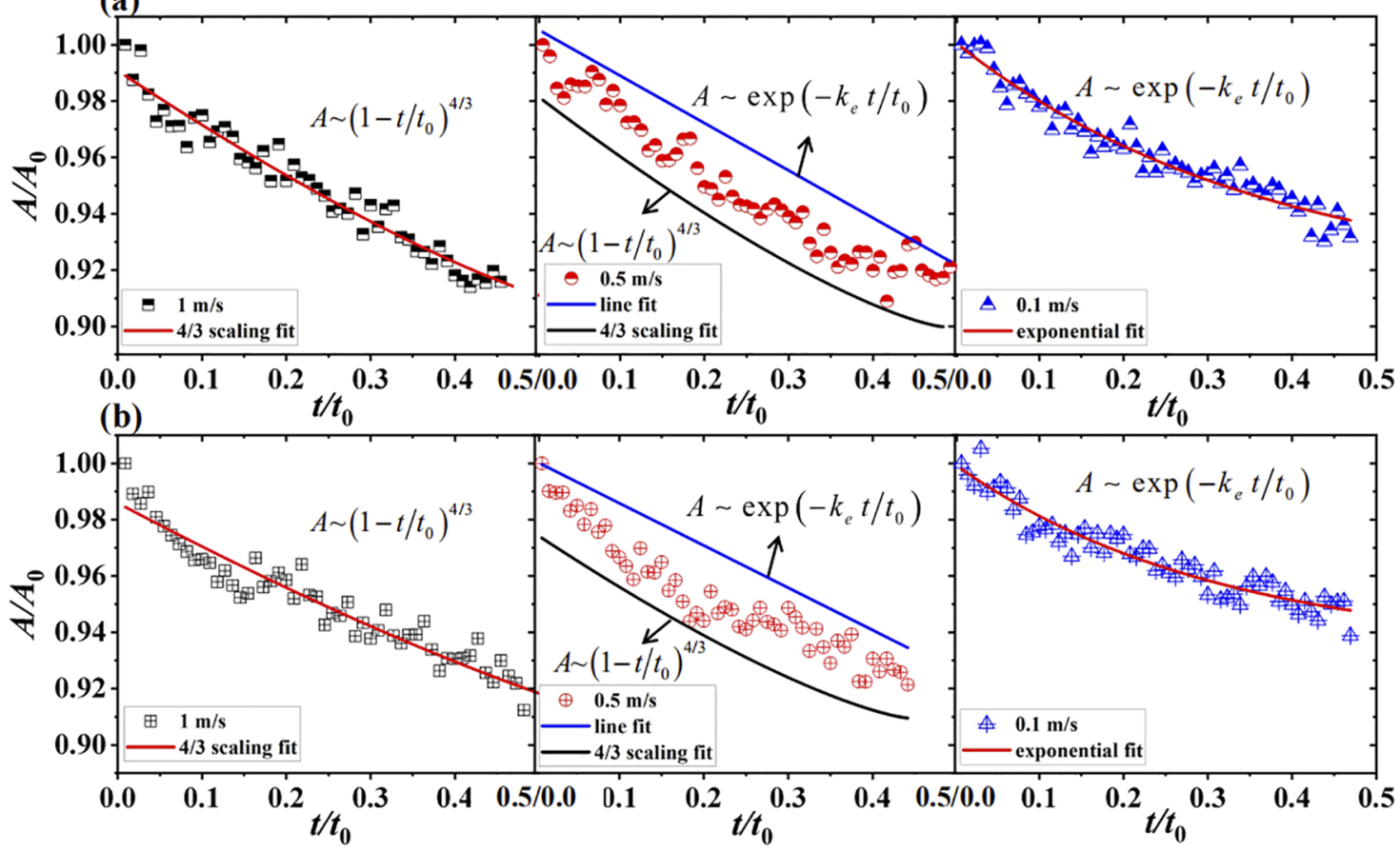

FIG. 7. Scaling laws of the solid area over time. From left to right: (a) half-filled black rectangles, red dots, and blue triangles are the statistical results of the cylinders when $D s$ is 4,2 , and 0.4 and (b) hollow black rectangles, red dots, and blue triangles are the statistical results for the rectangular prisms when $D s$ is 4,2 , and 0.4 , respectively. The red curves on the left and right and the black and blue lines in the middle correspond to the theoretical results.

\section{CONCLUSION}

The solid evolution in dissolutive flow is studied using MD simulations. The results show that final shapes are insensitive to the initial cross-sectional configurations due to the solid assembly at the nanoscale. We propose a dimensionless number $D s$ whose critical value controls the convergent state of the solid. When $0<D s$ $<1$, the soluble solid approaches a cylinder, and when $D s>1$, the solid section converges into a quasi-triangular prism. Nevertheless, the evolution processes of different initial configurations are not the same. The influences of atomic forces, size effects, and diffusion on the variability of the liquid density along different directions are discussed, and the fitting curve of the theoretical density distribution is given. Finally, the scaling laws that control the solid evolution with different $D s$ are described. Additional discussions in the future may involve the exact front angle size between different $D s$. We believe that our work provides a theoretical basis for structure optimization and industrial production.

\section{SUPPLEMENTARY MATERIAL}

See the supplementary material for the liquid density distribution and the behavior of dissolved particles from the simulation results.

\section{ACKNOWLEDGMENTS}

This work was jointly supported by the National Natural Science Foundation of China (NSFC; Grant Nos. 11722223, 11672300,
12072346, and 11872363), the Chinese Academy of Sciences Key Research Program of Frontier Sciences (Grant No. QYZDJ-SSWJSC019), and the Strategic Priority Research Program of the Chinese Academy of Sciences (Grant No. XDB22040401).

\section{DATA AVAILABILITY}

The data that support the findings of this study are available from the corresponding author upon reasonable request.

\section{REFERENCES}

${ }^{1}$ G. Marzun, H. Bönnemann, C. Lehmann, B. Spliethoff, C. Weidenthaler, and S. Barcikowski, "Role of dissolved and molecular oxygen on $\mathrm{Cu}$ and $\mathrm{PtCu}$ alloy particle structure during laser ablation synthesis in liquids," ChemPhysChem 18, 1175-1184 (2017).

${ }^{2}$ Y. P. Zhao, Nano and Mesoscopic Mechanics (Science Press, Beijing, 2014).

${ }^{3}$ J. Purseed, B. Favier, L. Duchemin, and E. W. Hester, "Bistability in RayleighBénard convection with a melting boundary," Phys. Rev. Fluids 5, 023501 (2020).

${ }^{4}$ H. Gomez, M. Bures, and A. Moure, "A review on computational modelling of phase-transition problems,” Philos. Trans. R. Soc., A 377, 20180203 (2019).

${ }^{\mathbf{5}}$ E. Hernandez, J. Otero, R. Santiago, R. Martinez, F. Castillo, and J. Oseguera, "Non parabolic interface motion for the 1-D Stefan problem: Dirichlet boundary conditions," Therm. Sci. 21, 2327-2336 (2017).

${ }^{6}$ M. Hadzic, G. Navarro, and S. Shkoller, "Local well-posedness and global stability of the two-phase Stefan problem," SIAM J. Math. Anal. 49, 4942-5006 (2017).

${ }^{7}$ Y. P. Zhao, Physical Mechanics of Surfaces and Interfaces (Science Press, Beijing, 2012).

${ }^{8}$ R. Raliya, T. Singh Chadha, K. Haddad, and P. Biswas, "Perspective on nanoparticle technology for biomedical use," Curr. Pharm. Des. 22, 2841 (2016). 
${ }^{9}$ J. J. Hobson, A. Al-Khouja, P. Curley, D. Meyers, C. Flexner, M. Siccardi, A. Owen, C. F. Meyers, and S. P. Rannard, "Semi-solid prodrug nanoparticles for long-acting delivery of water-soluble antiretroviral drugs within combination HIV therapies," Nat. Commun. 10, 1413 (2019).

${ }^{10}$ Y. Lan, Z. Yang, P. Wang, Y. Yan, L. Zhang, and J. Ran, “A review of microscopic seepage mechanism for shale gas extracted by supercritical $\mathrm{CO}_{2}$ flooding," Fuel 238, 412-424 (2019).

${ }^{11}$ Y. Xiao, J. Shao, S. K. Frape et al., "Groundwater origin, flow regime and geochemical evolution in arid endorheic watersheds: A case study from the Qaidam Basin, northwestern China," Hydrol. Earth Syst. Sc. 22, 4381 (2018).

${ }^{12}$ M. N. J. Moore, L. Ristroph, S. Childress, J. Zhang, and M. J. Shelley, "Selfsimilar evolution of a body eroding in a fluid flow," Phys. Fluids 25, 116602 (2013).

${ }^{13}$ P. J. Missel, L. E. Stevens, and J. W. Mauger, "Reexamination of convective diffusion/drug dissolution in a laminar flow channel: Accurate prediction of dissolution rate," Pharm. Res. 21, 2300-2306 (2004).

${ }^{14}$ D. Smrčka, J. Dohnal, and F. Štěpánek, "Dissolution and disintegration kinetics of high-active pharmaceutical granules produced at laboratory and manufacturing scale," Eur. J. Pharm. Biopharm. 106, 107-116 (2016).

${ }^{15}$ D. M. Anderson and M. G. Worster, "Weakly nonlinear analysis of convection in mushy layers during the solidification of binary alloys," J. Fluid Mech. 302, 307-331 (1995).

${ }^{16}$ I. McCue, A. Karma, and J. Erlebacher, "Pattern formation during electrochemical and liquid metal dealloying," MRS Bull. 43, 27-34 (2018).

${ }^{17}$ A. Singh, K. R. Ansari, D. S. Chauhan, M. A. Quraishi, H. Lgaz, and I. M. Chung, "Comprehensive investigation of steel corrosion inhibition at macro/micro level by ecofriendly green corrosion inhibitor in $15 \% \mathrm{HCl}$ medium," J. Colloid Interface Sci. 560, 225-236 (2020)

${ }^{18}$ C. Cohen, M. Berhanu, J. Derr, and S. C. du Pont, "Erosion patterns on dissolving and melting bodies," Phys. Rev. Fluids 1, 050508 (2016).

${ }^{19}$ E. Nakouzi, R. E. Goldstein, and O. Steinbock, "Do dissolving objects converge to a universal shape," Langmuir 31, 4145-4150 (2014).

${ }^{20}$ J. M. Huang, M. N. J. Moore, and L. Ristroph, "Shape dynamics and scaling laws for a body dissolving in fluid flow," J. Fluid Mech. 765, R3 (2015).

${ }^{21}$ M. N. J. Moore, "Riemann-Hilbert problems for the shapes formed by bodies dissolving, melting, and eroding in fluid flows," Commun. Pure Appl. Math. 70, 1810-1831 (2017).

${ }^{22}$ S. De and N. R. Aluru, "Energy dissipation in fluid coupled nanoresonators: The effect of phonon-fluid coupling," ACS Nano 12, 368-377 (2018).

${ }^{23}$ A. M. Rahmani, Y. Shao, M. Jupiterwala, and C. E. Colosqui, "Nanoscale flow past a colloidal cylinder confined in a slit channel: Lubrication theory and molecular dynamics analysis," Phys. Fluids 27, 082004 (2015).

${ }^{24}$ S. Sarkar, S. Ganguly, G. Biswas, and P. Saha, "Effect of cylinder rotation during mixed convective flow of nanofluids past a circular cylinder," Comput. Fluids 127, $47-64$ (2016).
${ }^{25} \mathrm{~K}$. Landry and N. Eustathopoulos, "Dynamics of wetting in reactive metal/ ceramic systems: Linear spreading,” Acta Mater. 44, 3923-3932 (1996).

${ }^{26}$ J. Yang, Q. Yuan, and Y.-P. Zhao, "Evolution of the interfacial shape in dissolutive wetting: Coupling of wetting and dissolution," Int. J. Heat Mass Transfer 118, 201-207 (2018).

${ }^{27}$ T. J. Singler, S. Su, L. Yin, and B. T. Murray, "Modeling and experiments in dissolutive wetting: A review," J. Mater. Sci. 47, 8261-8274 (2012).

${ }^{28} \mathrm{Q}$. Miao, Q. Yuan, and Y. P. Zhao, "Dissolutive flow in nanochannels: Transition between plug-like and Poiseuille-like," Microfluid. Nanofluid. 22, 141 (2018).

${ }^{29} \mathrm{P}$. A. Thompson and S. M. Troian, "A general boundary condition for liquid flow at solid surfaces," Nature 389, 360 (1997).

${ }^{30} \mathrm{P}$. Alipour, D. Toghraie, A. Karimipour, and M. Hajian, "Modeling different structures in perturbed Poiseuille flow in a nanochannel by using of molecular dynamics simulation: Study the equilibrium," Physica A 515, 13-30 (2019).

${ }^{31}$ D. C. Rapaport and E. Clementi, "Eddy formation in obstructed fluid flow: A molecular-dynamics study,” Phys. Rev. Lett. 57, 695 (1986).

${ }^{32}$ H. J. C. Berendsen, J. R. Grigera, and T. P. Straatsma, "The missing term in effective pair potentials," J. Phys. Chem. 91, 6269-6271 (1987).

${ }^{33}$ F. W. Straatsma, J. K. Nielsen, and H. E. Stanley, "Hydrogen-bond dynamics for the extended simple point-charge model of water," Phys. Rev. E 62, 579 (2000).

${ }^{34}$ T. Werder, J. H. Walther, R. L. Jaffe, T. Halicioglu, F. Noca, and P. Koumoutsakos, "Molecular dynamics simulation of contact angles of water droplets in carbon nanotubes," Nano Lett. 1, 697-702 (2001).

${ }^{35}$ P. G. De Gennes, "Wetting: Statics and dynamics," Rev. Mod. Phys. 57, 827 (1985).

${ }^{36} \mathrm{~N}$. Wu, L. Zeng, T. Fu, Z. Wang, and C. Lu, "Molecular dynamics study of rapid boiling of thin liquid water film on smooth copper surface under different wettability conditions," Int. J. Heat Mass Transfer 147, 118905 (2020).

${ }^{37}$ M. S. D. Wykes, J. Mac Huang, G. A. Hajjar, and L. Ristroph, "Self-sculpting of a dissolvable body due to gravitational convection," Phys. Rev. Fluids 3, 043801 (2018).

${ }^{38}$ M. H. Köhler, L. B. Da Silva, "Size effects and the role of density on the viscosity of water confined in carbon nanotubes," Chem. Phys. Lett. 645, 38-41 (2016).

${ }^{39}$ X. Chen, G. Cao, A. Han, V. K. Punyamurtula, L. Liu, P. J. Culligan, T. Kim, and Y. Qiao, "Nanoscale fluid transport: Size and rate effects," Nano Lett. 8, 2988-2992 (2008).

${ }^{40}$ S. Chen, N. Phan-Thien, B. C. Khoo, and X. J. Fan, "Flow around spheres by dissipative particle dynamics," Phys. Fluids 18, 103605 (2006).

${ }^{41} \mathrm{~N}$. Israelachvili, Intermolecular and Surface Forces (Academic Press, 2015).

${ }^{42}$ M. Doi, Soft Matter Physics (Oxford University Press, 2013).

${ }^{43} \mathrm{~B}$. Derjaguin and E. Obuchov, "Ultramicrometric analysis of solvate layer and elementary expansion effects," Acta Physicochim. URSS. 5, 1-22 (1936).

${ }^{44}$ A. Dokoumetzidis and P. Macheras, "A century of dissolution research: From Noyes and Whitney to the biopharmaceutics classification system," Int. J. Pharm. 321, 1-11 (2006). 